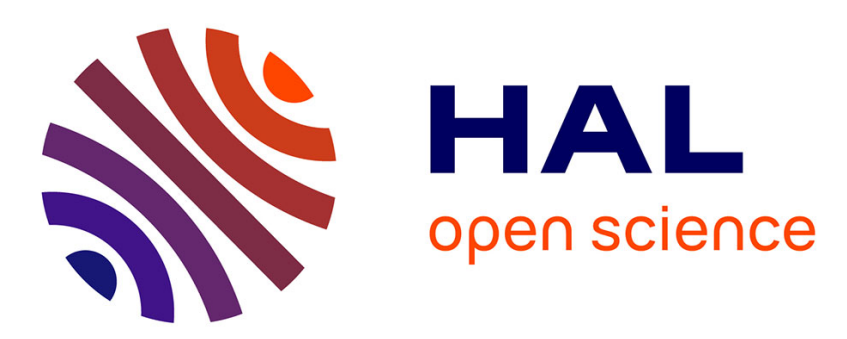

\title{
Unified preview control for humanoid postural stability and upper-limb interaction adaptation
}

\author{
Aurélien Ibanez, Philippe Bidaud, Vincent Padois
}

\section{To cite this version:}

Aurélien Ibanez, Philippe Bidaud, Vincent Padois. Unified preview control for humanoid postural stability and upper-limb interaction adaptation. 2012 IEEE/RSJ International Conference on Intelligent Robots and Systems, Oct 2012, Vilamoura, Portugal. To appear. hal-00720746

\section{HAL Id: hal-00720746 \\ https://hal.science/hal-00720746}

Submitted on 25 Jul 2012

HAL is a multi-disciplinary open access archive for the deposit and dissemination of scientific research documents, whether they are published or not. The documents may come from teaching and research institutions in France or abroad, or from public or private research centers.
L'archive ouverte pluridisciplinaire HAL, est destinée au dépôt et à la diffusion de documents scientifiques de niveau recherche, publiés ou non, émanant des établissements d'enseignement et de recherche français ou étrangers, des laboratoires publics ou privés. 


\title{
Unified preview control for humanoid postural stability and upper-limb interaction adaptation
}

\author{
Aurelien Ibanez, Philippe Bidaud and Vincent Padois
}

\begin{abstract}
This paper proposes a robust whole-body control formulation for biped balance in disturbed conditions by manipulation tasks. In order to include the effects of the interaction of the robot with its environment, required by the manipulation task in the balance control, we introduce a distributed preview control which captures both balance and manipulation behaviors and enables the regulation of the interaction impedance. The initial ZMP preview control is extended to take into account the disturbance resulting from the manipulation task and the preview control of adaptive impedances used to drive the upperlimbs. The resulting behavior is illustrated in a simple scenario. Its aptitude to dynamically extract an optimal control strategy improving tracking performances of both manipulation and balance tasks is also assessed when complex perturbations have to be compensated.
\end{abstract}

\section{INTRODUCTION}

To achieve using a humanoid robot interaction tasks in complex and dynamic environments such as those proposed for instance in the DARPA challenge [1], their control must be able to satisfy postural equilibrium while ensuring manipulation tasks to be performed under the best possible conditions. Maintaining the balance by the pressure of feet on the ground requires a reactive postural activity in response to disturbance. To optimize motor performances, the central nervous system in humans generates an Anticipatory Postural Adjustments (APA) thereby minimizing the effects of focal activity on postural balance. Conversely, motor activity related to the task adapts to constraints related to postural balance. The optimization of the overall performance implies a particular organization of the motor response with the aim to effectively coordinate the APAs and the focal activity [2].

One might be tempted to translate these principles observed in humans to control whole-body movement coordination of humanoid robots under external perturbations. From a mechanical point of view, they can be seen as parallel coupled sub-systems (see Fig. 1). The upper-limbs can be considered as adjustable mechanical impedances connected to an under-actuated mechanical platform sustentated on the ground by unilateral joints in a dynamic equilibrium through the lower-limbs motions. Motions of these subsystems induce mutual perturbations [3] and their behavior is affected by the interaction dynamics. The control issue of these interacting sub-systems having coupled dynamics and decoupled constraints and objectives can be addressed using a distributed model predictive control (DMPC) technique [4].

A. Ibanez, P. Bidaud and V. Padois are with Université Pierre et Marie Curie, Institut des Systèmes Intelligents et de Robotique, CNRS UMR 7222, 75005 Paris, France $\{$ ibanez, bidaud, padois $\}$ at isir.upmc.fr

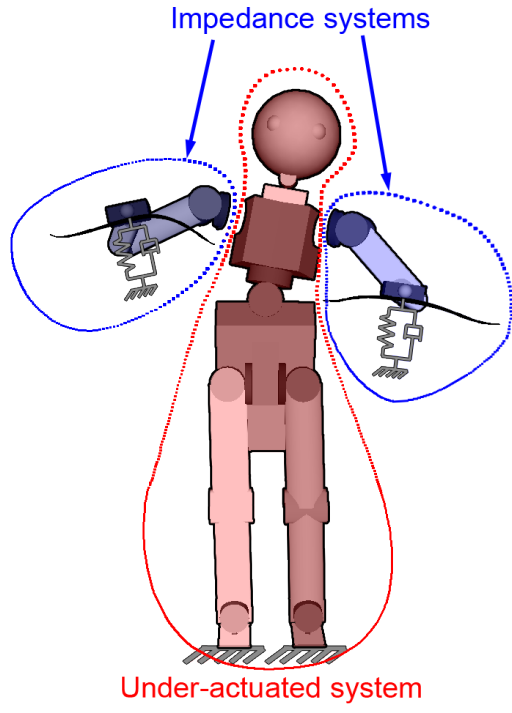

Fig. 1. System decomposition: Under-actuated postural system and upperlimbs impedance subsystems

Different frameworks for humanoid whole body motions control in multiple contact conditions have been proposed in a recent past using approaches basically relying on task prioritization [5] or ponderation [6].

Preview control presents two major advantages: it prepares the system for oncoming disturbances and counteracts the delays in the reaction of the system and distributed preview control [7] is an alternative which offers a way to coordinate local control inputs of sub-systems using decoupled local objectives. Preview control has been widely used in the particular field of active vehicle suspension in the work of Bender [8] and can be found under more complex formulations for instance in the work of Nakamura et al. [9] for the control of redundant manipulators and even for biped locomotion.

Safe biped walking has indeed encountered several improvements in the early 2000 s with a noticeable breakthrough from Kajita et al. [10] works on ZMP preview control. Computationally efficient methods to implement such control schemes can be derived for locomotion [11] and for wholebody motions [12], [13]. Extreme scenarii such as clash avoidance failure requiring robust balance recovery served as a fruitful context for the derivation of stable controllers. Kanzaki et al. [14] introduced a disturbance preview control to generate bracing motions by controlling future center of mass (CoM) trajectory, online previewing mainly resulting from stereo-vision and off line information (impact condi- 
tions). Other possible formulations are shown in different control frameworks: Morisawa et al. [15] presented a disturbance suppression control law with external force feedback; Prahlad et al. [16] used ankles joints to compensate the resulting torque of an external push.

In this context the disturbance is not directly applied to the center of mass of the robot; a model of the behavior of the whole-system under external disturbance, and notably its impedance, is hence needed. The awareness of the impedant behavior of any mechanical system in interaction grew in the mid 80s, leading to impedance control laws [17] and optimal impedance definitions [18], [19] in the operational space [20]. Predictive formulations [22] lead to unified controllers foreseeing changes in interaction dynamics and smoothly adjusting control inputs. Under this awareness this work proposes an approach to represent, adapt and preview the interaction of the robot with its environment in the manipulation task execution.

The approach presented in this work consists in developing a whole-body controller allowing for not only achieved biped balance but performant manipulation, through a distributed preview control formulation.

Robust control laws may allow for successful task execution subject to unknown disturbances; however, accounting for known or preview-able behaviors is expected to bring a significant increase in the performance of this execution. The hypothesis of known disturbance profile is strong; nevertheless, in a wide variety of cases the external disturbance results from voluntarily interacting with the environment: a future disturbance profile can be estimated and, as the contact is being set up, force measurements at the effector can provide sufficient information to build an estimated preview profile. To assess the contribution of the formulation introduced in this work, the future disturbance is assumed to be fully known.

This paper focuses on the simultaneous execution of two of the most generic tasks a humanoid robot can have to perform: manipulation and biped balance. The aim is hence at building distributed control laws accounting for known disturbances through their effect on the global system behavior; that is, the performance of the concurrent tasks executed. The subjection of the system is reduced in order to minimize or even take advantage of the effects of external disturbances by allowing the tuning of the tasks relevant parameters under a preview control scheme.

In this paper, the dynamic adaptation of the impedance manipulation is achieved along with the corresponding center of mass input to reduce the influence of the external disturbance in both balance and manipulation performance.

Contributions of this paper are:

- Inner and indirect accountancy of external disturbances resulting from manipulation in ZMP preview control through manipulation impedance.
- Definition of a task-oriented whole-body preview model coupling manipulation and balance controlled models and its performance evaluation function.

- Formulation of the corresponding extended controller optimizing the evaluation function by tuning relevant tasks parameters.

- Validation of the controllers through the simulation of highly disturbed scenarii.

\section{ZMP PREVIEW CONTROL AND COM DisturbANCE}

Notations are first introduced through the recall of the ZMP preview control method, that is extended later to account for center of mass disturbances.

Vectors and matrices are written in bold letters, $\dot{a}$ denotes the time derivative of $a$ and $\mathbf{A}^{T}$ is the transpose of $\mathbf{A}$. A cartesian vector $\mathbf{a}$ is written $\mathbf{a} \equiv\left[\begin{array}{lll}a_{x} & a_{y} & a_{z}\end{array}\right]^{T}$, where $(x, y)$ is the ground plane.

\section{A. ZMP Preview Control}

The works of Kajita et al. as described in [10] can be synthesized with the following system behavior description

$$
\begin{aligned}
\hat{\mathbf{x}}_{\mathbf{c}}(k+1) & =\mathbf{A} \hat{\mathbf{x}}_{\mathbf{c}}(k)+\mathbf{B u}(k) \\
\mathbf{p}(k) & =\mathbf{C} \hat{\mathbf{x}}_{c}(k)
\end{aligned}
$$

where $\mathbf{x}_{c}$ is the Center of Mass (CoM) cartesian position, $z_{c}$ the height of the CoM, $\mathbf{p}$ the ZMP position in the ground plane, $\mathbf{u}=\frac{\partial}{\partial t} \ddot{\mathbf{x}}_{c}$ the balance task input, $g$ the gravity acceleration and

$$
\begin{gathered}
\hat{\mathbf{x}}_{\mathbf{c}}(k) \equiv\left[\mathbf{x}_{c}(k d t) \dot{\mathbf{x}}_{c}(k d t) \ddot{\mathbf{x}}_{c}(k d t)\right]^{T}, \\
\mathbf{u}(k) \equiv \mathbf{u}(k d t), \mathbf{p}(k) \equiv \mathbf{p}(k d t), \\
\mathbf{A}=\left[\begin{array}{ccc}
1 & d t & d t^{2} / 2 \\
0 & 1 & d t \\
0 & 0 & 1
\end{array}\right], \mathbf{B}=\left[\begin{array}{c}
d t^{3} / 6 \\
d t^{2} / 2 \\
d t
\end{array}\right], \\
\mathbf{C}=\left[\begin{array}{lll}
1 & 0 & -z_{c} / g
\end{array}\right] .
\end{gathered}
$$

We then aim at minimizing at each step $k$ the objective function $f$ written as follows

$$
f(k)=\frac{1}{2} \sum_{i=k}^{\infty}\left[Q_{e} e_{p}(i)^{2}+\Delta \mathbf{x}^{T}(i) \mathbf{Q}_{x} \Delta \mathbf{x}(i)+R u^{2}(i)\right]
$$

where $e_{p}(i)=\left\|\mathbf{p}(i)-\mathbf{p}^{\text {ref }}(i)\right\|$ is the ZMP tracking error at step $i$ and $Q_{e}, \mathbf{Q}_{x}$ and $R$ are weights regulating the relative influence of the objective terms.

Wieber proposes in [11] to build the following hyperproblem by spreading Eqs. (1) and (2) on the finite horizon $\mathrm{N}$

$$
\left[\begin{array}{c}
\mathbf{p}(k) \\
\vdots \\
\mathbf{p}(k+N)
\end{array}\right]=\mathbf{P}_{\mathbf{x}} \hat{\mathbf{x}}_{\mathbf{c}}(k)+\mathbf{P}_{\mathbf{u}}\left[\begin{array}{c}
\mathbf{u}(k) \\
\vdots \\
\mathbf{u}(k+N)
\end{array}\right]
$$


where

$$
\begin{gathered}
\mathbf{P}_{\mathbf{x}}=\left[\begin{array}{ccc}
1 & d t & d t^{2} / 2-z_{c} / g \\
\vdots & \vdots & \vdots \\
1 & N d t & N^{2} d t^{2} / 2-z_{c} / g
\end{array}\right], \\
\mathbf{P}_{\mathbf{u}}=\left[\begin{array}{cccc}
\frac{d t^{3}}{6}-d t z_{c} / g & 0 & \cdots & 0 \\
\vdots & * & \ddots & 0 \\
\left(1+3 N+3 N^{2}\right) \frac{d t^{3}}{6}-d t \frac{z_{c}}{g} & \cdots & \cdots & *
\end{array}\right],
\end{gathered}
$$

that is rewritten, for the sake of simplicity,

$$
\hat{\mathbf{p}}_{k}=\mathbf{P}_{\mathbf{x}} \hat{\mathbf{x}}_{\mathbf{c}}(k)+\mathbf{P}_{\mathbf{u}} \hat{\mathbf{u}}_{k} .
$$

Hence Kajita's objective function in Eq. (4), with $\mathbf{Q}_{x}$ null, becomes

$$
f=\frac{1}{2} Q_{e}\left(\hat{\mathbf{p}}_{k}-\hat{\mathbf{p}}_{k}^{r e f}\right)^{2}+\frac{1}{2} R \hat{\mathbf{u}}_{k}^{2} .
$$

The pertinence of $f$ as an objective function requiring $Q_{e}$ and $R$ as positives, optimal horizon of input $\hat{\mathbf{u}}_{k}$ at step $k$ simply solves

$$
Q \mathbf{P}_{\mathbf{u}}^{T}\left(\mathbf{P}_{\mathbf{x}} \hat{\mathbf{x}}_{c}(k)+\mathbf{P}_{\mathbf{u}} \hat{\mathbf{u}}_{k}-\hat{\mathbf{p}}_{k}^{r e f}\right)+R \hat{\mathbf{u}}_{k}=\mathbf{0},
$$

from what we deduce

$$
\hat{\mathbf{u}}_{k}=-\left(\mathbf{P}_{\mathbf{u}}{ }^{T} \mathbf{P}_{\mathbf{u}}+\frac{R}{Q} I_{N \times N}\right)^{-1} \mathbf{P}_{\mathbf{u}}{ }^{T}\left(\mathbf{P}_{\mathbf{x}} \hat{\mathbf{x}}_{\mathbf{c}}(k)-\hat{\mathbf{p}}_{k}^{r e f}\right) .
$$

Previous works show that focusing on CoM behavior is sufficient to derive robust control laws ensuring safe biped balance. Therefore a formulation introducing external disturbances at the CoM level is first established, similar to Kajita's zero-moment point (ZMP) preview control, consisting in the tracking of a horizon of disturbed ZMP reference. Such a formulation can account for various types of disturbances, for instance acceleration variations occurring in vehicles.

\section{B. Center of Mass disturbance}

In the case of a disturbance $\mathbf{F}^{\text {dis }}$ acting directly on the $\mathrm{CoM}$ of the robot, the ZMP problem can be rewritten

$$
\boldsymbol{\tau}_{Z M P} \equiv\left[\begin{array}{c}
\text { Find p such that } \\
-M g \delta_{y}+M \ddot{\mathbf{x}}_{c_{y}} z+\left(\boldsymbol{\delta} \times \mathbf{F}^{d i s}\right)_{x} \\
M g \delta_{x}-M \ddot{\mathbf{x}}_{c_{x}} z+\left(\boldsymbol{\delta} \times \mathbf{F}^{d i s}\right)_{y} \\
\delta_{x} M \ddot{\mathbf{x}}_{c_{y}}-\delta_{y} M \ddot{\mathbf{x}}_{c_{x}}+\left(\boldsymbol{\delta} \times \mathbf{F}_{d i s}\right)_{z}
\end{array}\right]=\mathbf{0}
$$

where $M$ is the total mass of the system, $\boldsymbol{\delta} \equiv\left[\begin{array}{c}\left.\mathbf{x}_{c}\right|_{x y}-\mathbf{p} \\ z_{c}-0\end{array}\right]$ and $\left.\mathbf{x}_{c}\right|_{x y}$ the projection of $\mathbf{x}_{c}$ in the ground plane. Eq. (2) now writes

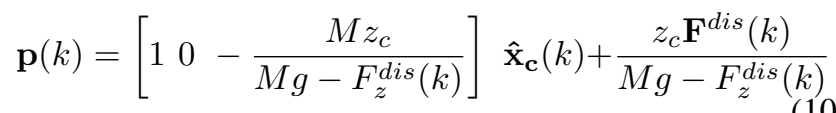

and $\alpha_{0}^{d i s} \equiv \frac{1}{M g-F_{z}^{d i s}}, \alpha^{d i s} \equiv \frac{\mathbf{F}^{d i s}}{M g-F_{z}^{d i s}}$ are used to rewrite Eq. (6) as

$$
\mathbf{p}_{k}=\mathbf{P}_{\mathbf{x}}^{\text {dis }} \hat{\mathbf{x}}_{\mathbf{c}}(k)+\mathbf{P}_{\mathbf{u}}^{\text {dis }} \hat{\mathbf{u}}_{k}+\mathbf{D}_{k}
$$

where

$$
\begin{gathered}
\mathbf{P}_{\mathbf{x}}^{\text {dis }}=\left[\begin{array}{cccc}
1 & d t & d t^{2} / 2-M z_{c} \alpha_{0}^{d i s}(k) & \\
\vdots & \vdots & \vdots \\
1 & N d t & N^{2} d t^{2} / 2-M z_{c} \alpha_{0}^{d i s}(k+N)
\end{array}\right]_{(12)} \\
\mathbf{P}_{\mathbf{u}}^{\text {dis }}=\left[\begin{array}{ccc} 
\\
d t^{3} / 6-d t M z_{c} \alpha_{0}^{d i s}(k) & \cdots & 0 \\
\vdots & \ddots & 0 \\
\left(1+3 N^{2}\right) d t^{3} / 6-d t M z_{c} \alpha_{0}^{d i s}(k+N) & \cdots & *
\end{array}\right]
\end{gathered}
$$

and finally

$$
\mathbf{D}_{k}=\left[\begin{array}{lll}
z_{c} \alpha^{d i s}(k) & \cdots & z_{c} \alpha^{d i s}(k+N)
\end{array}\right]^{T} .
$$

Optimal horizon of solution CoM input follows

$\hat{\mathbf{u}}_{k}=-\left(\mathbf{P}_{\mathbf{u}}^{\mathbf{d i s} T} \mathbf{P}_{\mathbf{u}}^{\mathrm{dis}}+\frac{R}{Q} I_{N \times N}\right)^{-1} \mathbf{P}_{\mathbf{u}}^{\mathrm{dis}}{ }^{T}\left(\mathbf{P}_{\mathbf{x}}^{\mathrm{dis}} \mathbf{x}_{\mathbf{c}}(k)-\hat{\mathbf{p}}_{k}^{r d}\right)$

where

$$
\hat{\mathbf{p}}_{k}^{r d} \equiv \hat{\mathbf{p}}_{k}^{r e f}-\mathbf{D}_{k}
$$

that can be interpreted as a derivate horizon $\hat{\mathbf{p}}^{r d}$ of disturbed ZMP reference.

A straightforward way to account for external disturbances acting directly on the CoM of the system in the ZMP Preview Control Method is introduced in this section. Hence, from a reduced approach of the controlled effector behavior, a way to deal with disturbances acting on the effector itself at the CoM level is proposed in the following section.

\section{ZMP PREVIEW CONTROL WITH EQUIVALENT COM DISTURBANCE}

In this section a coupled reduced model describing both balance and manipulation behaviors is built. It is referred to as a previewer, task-oriented as it encapsulates the controlled whole-body dynamics. From this previewer transmitted external disturbance to the CoM can be extracted to build a disturbed controller similarly to the previous section. However the problem can be augmented to develop an extended controller tuning manipulation task parameters in order to achieve successful concurrent manipulation and balance in highly-disturbed contexts.

\section{A. Preliminaries - Effector behavior}

CoM disturbances only represent a limited variety of external actions. Indeed, high-value disturbances mainly come from - required or not - physical interaction with the environment, ie apply on a physical point. In the context of manipulation, this point is very likely to be the endeffector. This effector is the point where a manipulation task is described. In a position-control framework, an operational space control law of the following form is assumed

$$
\ddot{\mathbf{x}}^{c m d}=K^{p}\left(\mathbf{x}^{d e s}-\mathbf{x}\right)+K^{d}\left(\dot{\mathbf{x}}^{d e s}-\dot{\mathbf{x}}\right)+\ddot{\mathbf{x}}^{d e s},
$$

where $\mathbf{x}$ is the cartesian space position of the end-effector and $K^{p}, K^{d}$ proportional and derivative gains, respectively. 
In this framework, a LQP solver [6] computes the input joint torques $\boldsymbol{\tau}^{c m d}$ that provides $\ddot{\mathbf{q}}^{c m d}$ such that the error

$$
\left\|\ddot{\mathbf{x}}^{c m d}-\mathbf{J}_{e} \ddot{\mathbf{q}}^{c m d}+\dot{\mathbf{J}}_{e} \dot{\mathbf{q}}\right\|
$$

is minimized. In the joint space we write

$$
\boldsymbol{\tau}^{c m d} \text { such that } \mathbf{H} \ddot{\mathbf{q}}^{c m d}+\mathbf{N}+\mathbf{G}=\boldsymbol{\tau}^{c m d}+\mathbf{J}_{\text {cont }}^{T} \mathbf{F}_{\text {cont }}
$$

and the disturbed dynamics writes, assuming no configuration change,

$$
\mathbf{H} \ddot{\mathbf{q}}+\mathbf{N}+\mathbf{G}=\boldsymbol{\tau}^{c m d}+\mathbf{J}_{\text {cont }}^{T} \mathbf{F}_{\text {cont }}^{\prime}+\mathbf{J}_{e}^{T} \mathbf{F}_{\text {ext }},
$$

where $\mathbf{H}, \mathbf{N}$ and $\mathbf{G}$ are respectively the mass and centrifugal/Coriolis matrices, $\mathbf{G}$ the gravity vector, $\mathbf{J}_{\text {cont }}^{T}, \mathbf{F}_{\text {cont }}$ and $\mathbf{J}_{e}^{T}, \mathbf{F}_{\text {ext }}$ the jacobian and efforts at the contacts with the ground and at the effector, respectively.

Considering the main parts involved in the manipulation task, we isolate the partial mechanical system composed of the serial chain of bodies from the end-effector to an arbitrarily selected parent body (in this case, the chest), as shown as an example in Fig. 2, subject to the dynamics

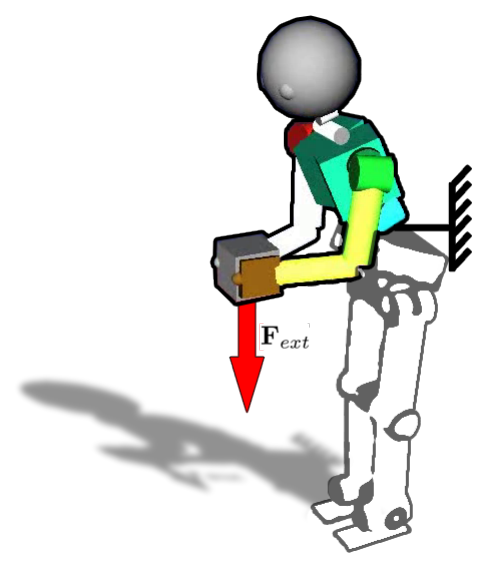

Fig. 2. Partial Dynamics: upper-limbs impedance system

$$
\tilde{\mathbf{H}} \ddot{\tilde{\mathbf{q}}}+\tilde{\mathbf{N}}+\tilde{\mathbf{G}}=\tilde{\boldsymbol{\tau}}^{c m d}+\mathbf{J}_{e}^{T} \mathbf{F}_{e x t}
$$

while Eq. (19) is simply written in this problem

$$
\tilde{\mathbf{H}} \ddot{\tilde{\mathbf{q}}}^{c m d}+\tilde{\mathbf{N}}+\tilde{\mathbf{G}}=\tilde{\boldsymbol{\tau}}^{c m d}
$$

The $\tilde{a}$ notation denotes the selected subset of $a$ due to the reduction of the problem dimensionality ${ }^{1}$.

From these two previous equations we can derive the dynamics of the disturbance resulting from an external force at the end-effector

$$
\begin{aligned}
\mathbf{M}_{e} \ddot{\mathbf{x}}= & \mathbf{F}_{e x t}-\mathbf{M}_{e} \dot{\mathbf{J}}_{e} \mathbf{J}_{e}^{\dagger}\left(\dot{\mathbf{x}}-\dot{\mathbf{x}}^{c m d}\right) \\
& +\mathbf{K}_{e}\left(\mathbf{x}^{d e s}-\mathbf{x}\right)+\mathbf{C}_{e}\left(\dot{\mathbf{x}}^{d e s}-\dot{\mathbf{x}}\right)+\mathbf{M}_{e} \ddot{\mathbf{x}}^{d e s}
\end{aligned}
$$

where

$$
\mathbf{M}_{e} \equiv\left(\mathbf{J}_{e} \tilde{\mathbf{H}}^{-1} \mathbf{J}_{e}^{T}\right)^{-1}, \mathbf{K}_{e} \equiv K^{p} \mathbf{M}_{e}, \mathbf{C}_{e} \equiv K^{d} \mathbf{M}_{e}
$$

\footnotetext{
${ }^{1}$ Note that, although the manipulation model is restricted to the upperbody dynamics, the control formulation providing input torques considers whole-body balance and manipulation.
}

and $\mathbf{J}_{e}^{\dagger}$ is the dynamically consistent pseudo-inverse of the Jacobian of the end-effector. Eq. (23) describes dynamics that we can synthesize in the model shown in Fig. 3, by writing $\mathbf{F}_{v e l}=-\mathbf{M}_{e} \dot{\mathbf{J}}_{e} \mathbf{J}_{e}^{\dagger}\left(\dot{\mathbf{x}}-\dot{\mathbf{x}}^{c m d}\right)$

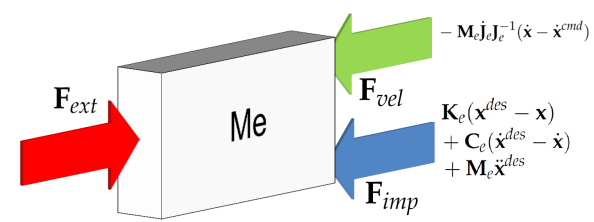

Fig. 3. Equivalent upper-limbs model: Manipulation previewer

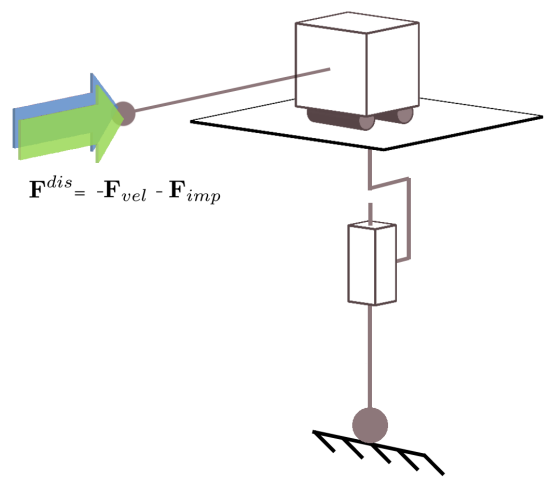

Fig. 4. Equivalent under-actuated system model: Balance previewer

which encapsulates operationnal effects of the task execution error resulting from the external disturbance, and $\mathbf{F}_{i m p}=\mathbf{K}_{e}\left(\mathbf{x}^{\text {des }}-\mathbf{x}\right)+\mathbf{C}_{e}\left(\dot{\mathbf{x}}^{\text {des }}-\dot{\mathbf{x}}\right)+\mathbf{M}_{e} \ddot{\mathbf{x}}^{\text {des }}$ standing for virtual effector impedance describing the control law.

External disturbances can now be accounted for at the CoM level of consideration and henceforth the formulation of Sec. II-B can be used in a more generic way.

\section{B. Coupled Behavior}

The manipulation model is concatenated with the inverted pendulum one to build the complete model composed of the manipulation and balance models shown in Figs. 3 and 4, respectively, which relies on the decoupling hypothesis described in Fig. 5. The transmission of external disturbance through the main mechanical chain involved in the manipulation task is described and the application of the resulting effort (that applies thoroughly to the rest of the body) is considered to occur at the center of mass of the robot (which is, of course, a non-physical point). The validity of this approximation decreases with high CoM accelerations, and a specific attention needs to hover the choice of the manipulation chain and of the dynamic parameters (mass, notably) involved in each description (upper/lower behavior).

This model is described over a preview horizon in the way Kajita et al. previewed the inverted pendulum behavior. The 


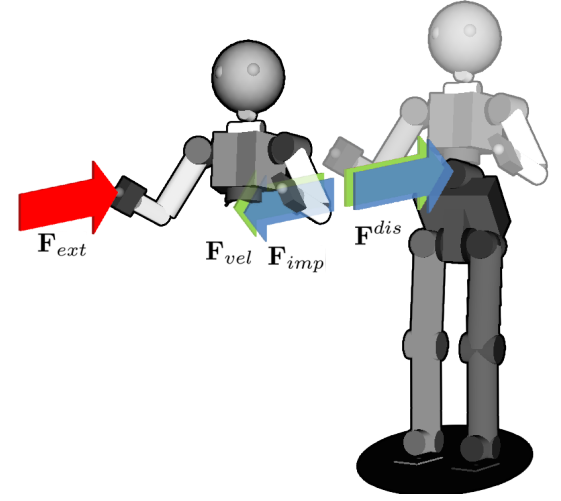

Fig. 5. Equivalent Global Model explicitation

controlled end-effector follows

$$
\begin{gathered}
\hat{\mathbf{x}}_{k+1}=\left[\begin{array}{ccc}
1 & d t & d t^{2} / 2 \\
0 & 1 & d t \\
-K_{k}^{p} & -d t K_{k}^{p}-K_{k}^{d} & -\frac{d t^{2}}{2} K_{k}^{p}-d t K_{k}^{d}
\end{array}\right] \hat{\mathbf{x}}_{k} \\
+\left[\begin{array}{c}
0 \\
0 \\
K_{k}^{p} \mathbf{x}_{k}^{d e s}+K_{k}^{d} \dot{\mathbf{x}}_{k}^{d e s}+\ddot{\mathbf{x}}_{k}^{d e s}+\left(\mathbf{J}_{e} \mathbf{H}^{-1} \mathbf{J}_{e}^{T}\right) \mathbf{F}_{k}^{e x t}
\end{array}\right] \\
+\left[\begin{array}{c}
0 \\
0 \\
+\dot{\mathbf{J}}_{e} \mathbf{J}_{e}^{\dagger}\left(\dot{\mathbf{x}}^{c m d}-\dot{\mathbf{x}}_{k}-d t \ddot{\mathbf{x}}_{k}\right)
\end{array}\right],
\end{gathered}
$$

while the CoM and ZMP positions remain described by

$$
\begin{aligned}
\hat{\mathbf{x}}_{k+1}^{\mathbf{c}} & =\mathbf{A} \hat{\mathbf{x}}_{k}^{\mathbf{c}}+\mathbf{B} \mathbf{u}_{k}, \\
\mathbf{p}_{k} & =\left[10-\frac{M z_{c}}{M g-\left.F_{k}^{d i s}\right|_{z}}\right] \hat{\mathbf{x}}_{k}^{\mathbf{c}}+\frac{z_{c} \mathbf{F}_{k}^{d i s}}{M g-\left.F_{k}^{d i s}\right|_{z}},
\end{aligned}
$$

where we express the CoM disturbance $\mathbf{F}_{\text {dis }}$ as follows:

$$
\begin{aligned}
\mathbf{F}_{k}^{d i s}= & \mathbf{M}_{e} \dot{\mathbf{J}}_{e} \mathbf{J}_{e}^{\dagger}\left(\dot{\mathbf{x}}_{k}-\dot{\mathbf{x}}_{k}^{c m d}\right)-\mathbf{M}_{e} \ddot{\mathbf{x}}_{k}^{d e s} \\
& -\mathbf{K}_{k}^{e}\left(\mathbf{x}_{k}^{d e s}-\mathbf{x}_{k}\right)-\mathbf{C}_{k}^{e}\left(\dot{\mathbf{x}}_{k}^{d e s}-\dot{\mathbf{x}}_{k}\right) .
\end{aligned}
$$

\section{Disturbed controller: Natural disturbance absorption}

We proceed precisely as described in Sec. II-B, except that CoM disturbance now implicitly accounts for external disturbance ( $c f$ Eq. (25)) and natural absorption by the manipulation task.

The disturbance occurring at the hand level is hence indirectly accounted for at the CoM level, providing more precise insight of the behavior of the disturbance against the manipulation control execution. However, no other use than the extraction of relevant information is made of the consideration of the manipulation impedance. $A 1$ will denote this formulation in the following sections.

To take advantage of this consideration the possibility to adjust the impedance of the manipulation to adapt the whole-body dynamics to the disturbance is introduced in the following section.

\section{Extended Controller: Controlled disturbance absorption}

We propose to extend the optimization problem to the following, aiming at reducing the transmission of external disturbances by dynamically tuning the manipulation task gains along with the CoM control input.

The problem becomes:

Find optimal $\left(\mathbf{u}_{j}, K_{j}^{p}, K_{j}^{d}\right)_{j=k . k+N}$ that minimize

$$
\begin{aligned}
g_{k}= & \frac{1}{2} \sum_{i=k}^{k+N} Q_{e}\left(\mathbf{p}_{i}-\mathbf{p}_{i}^{r e f}\right)^{2}+Q_{t}\left(\hat{\mathbf{x}}_{i}-\hat{\mathbf{x}}_{i}^{\text {des }}\right)^{2} \\
& +R \mathbf{u}_{i}^{2}+S\left[\begin{array}{ll}
K_{i}^{p} & K_{i}^{d}
\end{array}\right]^{2}+T\left[\begin{array}{ll}
\Delta K_{i}^{p} & \Delta K_{i}^{d}
\end{array}\right]^{2}
\end{aligned}
$$

where $\Delta K_{i}^{p}=K_{i}^{p}-K_{i-1}^{p}$ and $Q_{e}, Q_{t}, R, S$ and $T$ are weights defining the relative influence between objective and regulating terms.

The variables $\left(\mathbf{u}_{j}, K_{j}^{p}, K_{j}^{d}\right)_{j=k . . k+N}$ appear as relevant parameters to tune: CoM control input $\mathbf{u}_{k}$ defines the future behavior of the center of mass of the robot to satisfy balance while $K_{k}^{p}$ and $K_{k}^{d}$ determine the manipulation impedance. Such an objective function simultaneously aims at minimizing the error on concurrent tasks execution while avoiding high changes in parameters. This extended controller is noted controller $C 2$ in the following sections.

\section{Simulated Results}

We propose to assess the validity of the reduced model introduced in the previous sections through the simulation of a humanoid system exposed to a known external disturbance while performing both manipulation and balance tasks.

Simulated experiments are performed using ArborisPython [23], an open-source dynamic simulator developed at ISIR with the Python programming language. The implementation of LQP relies on CVXOPT/CVXMOD, twofree Python packages dedicated to convex optimization [24]. The simulated robot is a rather accurate model of the iCub robot [25], 38 degrees of freedom are simulated $(32+6$ floating joints to locate the root in space), and 4 contact points at each foot.

The simulated scene is shown in Fig. 6: a controller governing the door joint applies a specific torque considered as known by the controller. The hand and the door are constrained by a spherical joint.

\section{A. First scenario: single impact}

The system is first exposed to the external disturbance shown on the left of Fig. 6. The same scenario is launched three times with a different controller at each round:

- PD controller and original Kajita-Wieber Controller ("controller C0"),

- PD controller and disturbed balance controller as described in Sec. III-C ("controller C1"),

- PD controller and whole-body extended controller presented in Sec. III-D ("controller C2").

This simple scenario provides a way to illustrate and follow the incremental building of the extended solution through successive and additive gains in performance. 


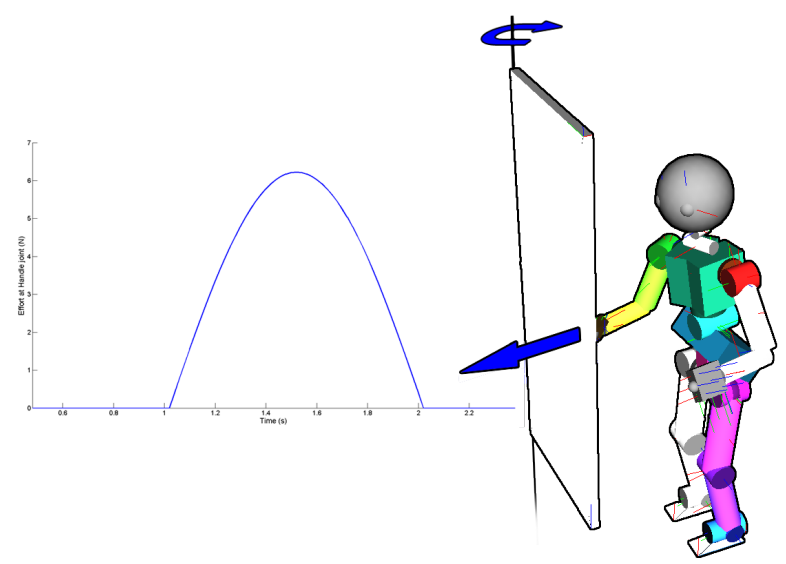

Fig. 6. Experimental setting: the humanoid must keep the door closed and maintain postural balance. The first scenario consists in the force profile applied at the hand shown on the left of the figure.

1) Safe balance: First of all, the consideration of the external disturbance in the balance task control leads to a significant increase in ZMP tracking performance, as shown in Fig. 7: effects of the impact on the ZMP position is notably ${ }^{2}$ reduced and allows the ZMP to stay within the sustentation hull, therefore ensuring stable balance. This gain in performance is not a direct consequence of the extension of the balance controller but of the enhanced balance controller.

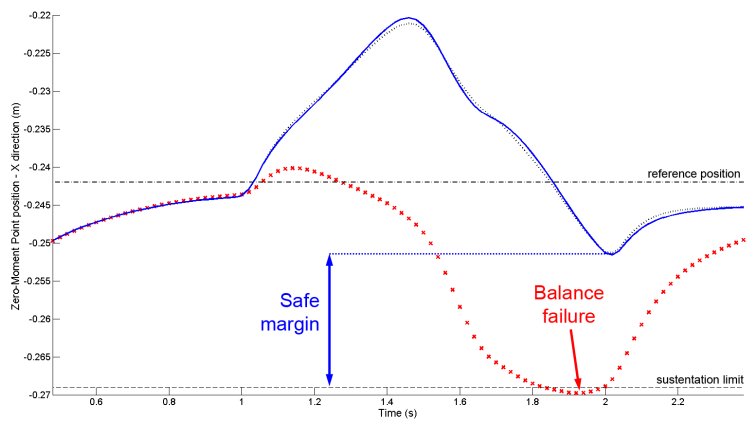

Fig. 7. First scenario: Zero-Moment Point position - dotted: controller $C 0$, crosses: $C 1$, solid: $C 2$

2) Performant manipulation: Second, in a single impact context, a robust balance resulting from the manipulation task behavior and external disturbance consideration provides a gain in the manipulation task tracking of around $10 \%$ compared to the average-equivalent constant impedance controller $C 0$ as Fig. 8 shows.

3) Impedance adaptation: The extended controller achieves the automatic extraction of a rather intuitive strategy (Fig. 9): it keeps a high rigidity for the first instants of impact to avoid outrageous manipulation tracking error, softens the impedance while the impact strengthens, recovers a high rigidity during impact fading and finally reduces the impedance to allow for easier balance recovery as no disturbance is expected to interfere.

\footnotetext{
${ }^{2}$ in the direction of the disturbance
}

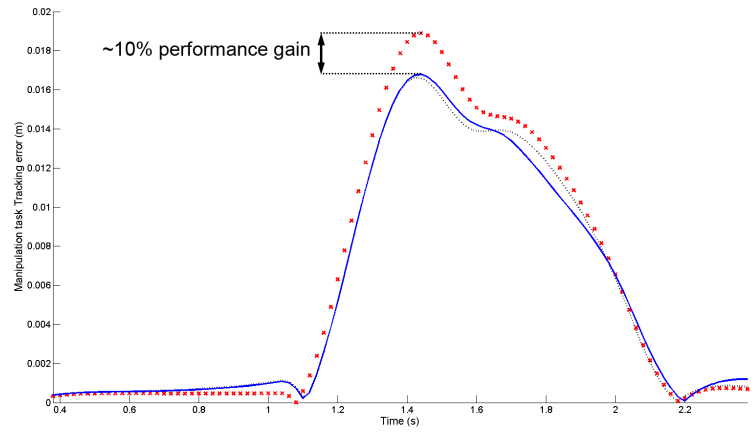

Fig. 8. First scenario: Manipulation tracking error - dotted: controller $C 0$, crosses: $C 1$, solid: $C 2$

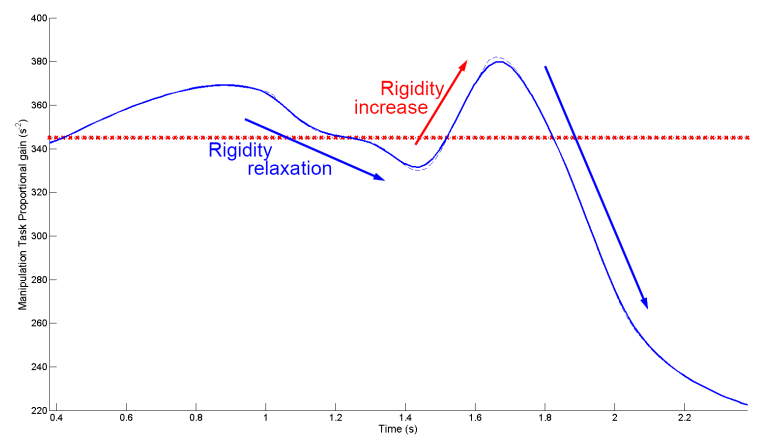

Fig. 9. First scenario: Controlled Proportional gain - dotted: controller $C 0$, crosses: $C 1$, solid: $C 2$

\section{B. Second scenario: multiple impacts}

We propose a second scenario to compare the three different controllers consisting in multiple impacts of different amplitudes (Fig. 10). This simulation brings out wider insights into the contribution of such controllers.

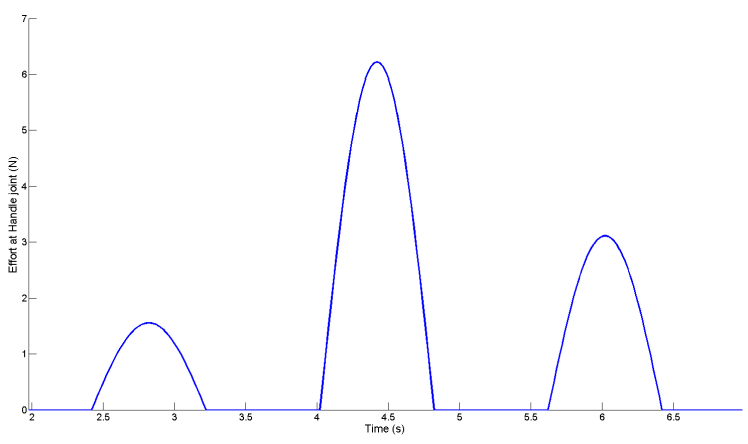

Fig. 10. Second scenario: Force applied at Handle constraint

1) Accounting for disturbance: The consideration of transmitted disturbance seems to cause a slight improvement in terms of manipulation tracking in case of high value impact ( $c f$ Fig. 11), as the overall state of the robot $^{3}$ is noticeably (and fortunately) less disturbed when external disturbance is accounted for (Fig. 12).

Data of higher relevance in this study shows the contribution of the disturbed controller: we notice in Fig. 13 that the ZMP

${ }^{3}$ Looked at through its center of mass. 


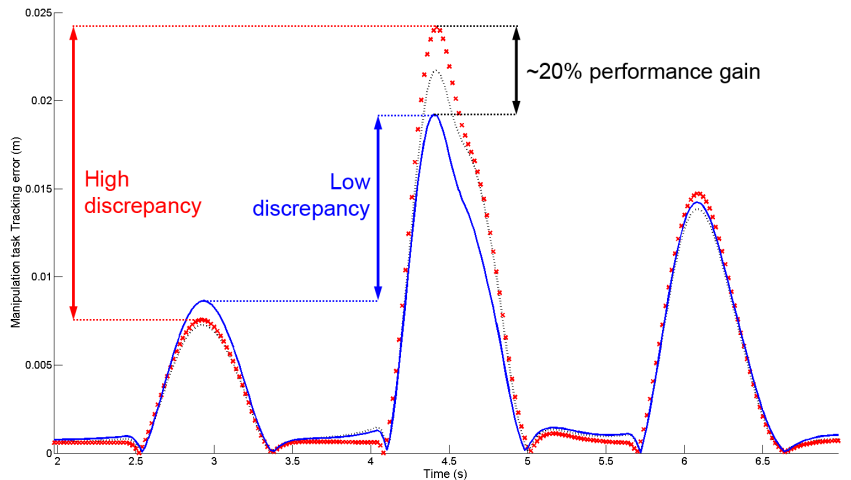

Fig. 11. Second scenario: Manipulation tracking error - dotted: controller $C 0$, crosses: $C 1$, solid: $C 2$

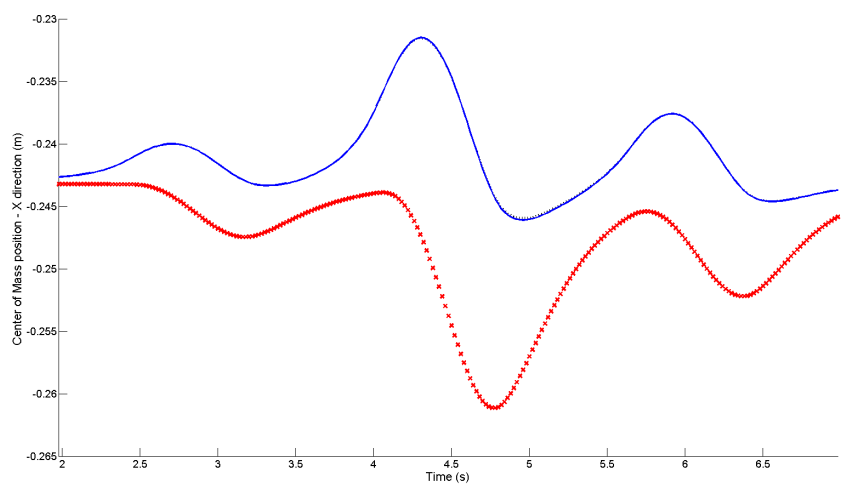

Fig. 12. Second scenario: Center of Mass position - dotted: controller $C 0$, crosses: $C 1$, solid: $C 2$

of the system stays within the ZMP reference bounds with such a controller, whereas Kajita's controller temporarily fails (in such conditions with $R / Q_{e}=5 . e^{-7}$ and a preview horizon of $1.5 s$ ) to hold the ZMP within the sustentation hull.

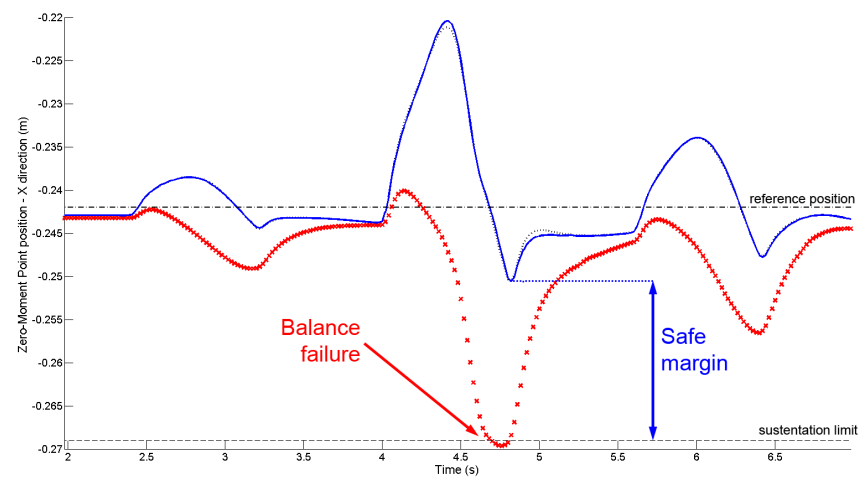

Fig. 13. Second scenario: Zero-Moment Point position - dotted: controller $C 0$, crosses: $C 1$, solid: $C 2$

The computational cost of controller $C 1$ is not relevantly higher than for $C 0$, as the dimensionality of the problem is not increased.

2) Gain-Controlled Manipulation task: This controller consists in finding an optimal horizon of manipulation task proportional gains and the corresponding optimal balance task input horizon in order to minimize tracking error on both tasks. A Nelder-Mead simplex algorithm [26] with warmstart is used over the feasible (manipulation task) proportional gains space for which optimal balance input (using the disturbed controller) is computed at each vertex and performance is evaluated. This algorithm is a naive procedure allowing to compute a pseudo-optimal solution, and adds a significant computational cost to controller $C 1$ (approx. $\mathrm{x} 10$ ). Although future works will focus on the solving of the augmented optimization problem, this algorithm provides sufficient insight in the contribution brought by controller $C 2$.

Similarly to the disturbed controller, it allows to reduce the overall system disturbance and to maintain balance. However, chosen parameters $Q_{t}$ and $Q_{e}$ implicitly define an admissible tracking error, and henceforth a slight loss in manipulation tracking for the first (and lowest) perturbation is seen.

As expected the obtained strategy of changes in proportional gain (Fig. 14) coupled to optimal input induces an automated regularization of both tracking tasks.

It provides a robust manipulation, less dependent on the disturbance intensity as a noticeably lower discrepancy in the manipulation task is observed through impacts of different values, along with a tracking performance increase for strong impacts ( $c f$ Fig. 11).

No prior estimation of the manipulation task gain is needed, the coupled controller tuning it according to perceived disturbances and behavior preview.

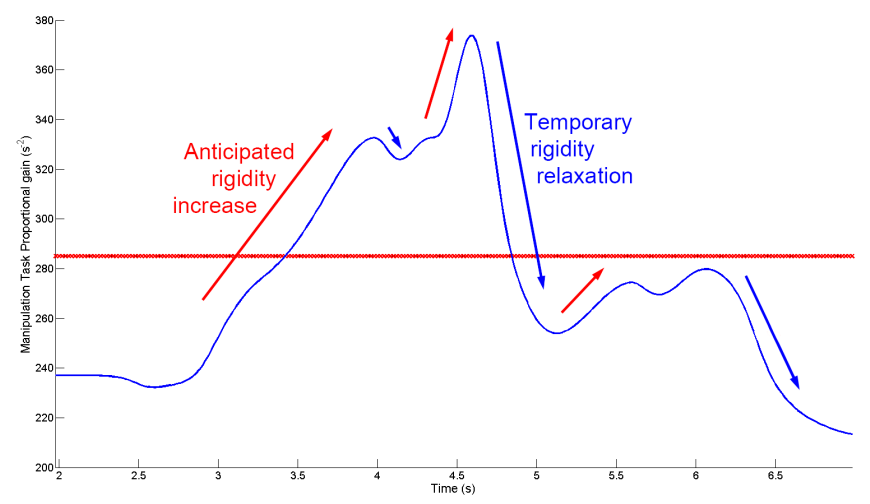

Fig. 14. Second scenario: Controlled Proportional gain - dotted: controller $C 0$, crosses: $C 1$, solid: $C 2$

\section{CONCLUSion}

The obvious need for the accountancy of known disturbances in critical controllers such as balance controllers has been illustrated. While it can provide higher performance, we cannot ignore the concurrency of the different tasks a robot can perform as disturbances propagate through the whole-body dynamics, dynamics that are subject to controlled actuators. The consideration of the coupling between simultaneous tasks can provide not only information to recover from high disturbances but solutions to effectively use this coupling to take advantage of such 
disturbances.

The introduction of a higher level extended controller shows indeed that control strategies can be extracted without costly planning or heuristic rules.

Efforts have been made to find ways to successfully perform several tasks simultaneously. Our effort is set to maximize the performance of this simultaneous execution: the main contribution of such a formulation is to go beyond the execution of a set of independent tasks to consider their effect on the robot dynamics and their behavior in disturbed condition as a whole.

We propose as future works the study of a different formulation: instead of controlling the apparent stiffness of the manipulating chain, we indirectly control internal forces by setting up new virtual objectives to the manipulation task. Such a formulation provides an algebraic resolution to the optimization problem though reducing the dimension of the controlled parameters set.

\section{ACKNOWLEDGMENT}

This work was partially supported by the City of Paris for the ANIPEV Project and by the RTE company through its chair "Robotics Systems for field intervention in constrained environments" hold by Vincent Padois.

\section{REFERENCES}

[1] “Darpa robotics challenge." [Online]. Available: http://go.usa.gov/mVj

[2] A. Wing, J. R. Flanagan, and J. Richardson, "Anticipatory postural adjustments in stance and grip," Experimental Brain Research, vol. 116, pp. 122-130, 1997.

[3] A. Albu-Schffer, C. Ott, U. Frese, and G. Hirzinger, "Cartesian impedance control of redundant robots: Recent results with the dlrlight-weight-arms," in Proceedings of the International Conference on Robotics and Automation, vol. 3, 2003, pp. 3704-3709.

[4] A. N. Venkat, "Distributed model predictive control : theory and applications," Ph.D. dissertation, 2006.

[5] L. Sentis and O. Khatib, "A whole-body control framework for humanoids operating in human environments," in Proceedings of the IEEE International Conference on Robotics \& Automation, 2006.

[6] J. Salini, V. Padois, and P. Bidaud, "Synthesis of complex humanoid whole-body behavior: a focus on sequencing and tasks transitions," in Proceedings of the IEEE International Conference on Robotics \& Automation, 2011.

[7] E. Camponogara, D. Jia, B. Krogh, and S. Talukdar, "Distributed model predictive control," IEEE Control Systems, vol. 22, pp. 44-52, 2002

[8] E. Bender, "Optimum linear preview control with application to vehicle suspension," Journal of Basic Engineering, vol. 90, no. 2, pp. 213-222, 1968.

[9] Y. Nakamura, Z. Tonkxiao, and M. Minami, "Multi-preview configuration control for predictive behavior of redundant manipulator," in ICCAS-SICE, 2009, pp. 3117-3123.

[10] S. Kajita, F. Kanehiro, K. Kaneko, K. Kajiwara, K. Harada, K. Yokoi, and $\mathrm{H}$. Hirukawa, "Biped walking pattern generation by using preview control of zero-moment point," in IEEE International Conference on Robotics \& Automation, 2003.

[11] P.-B. Wieber, "Trajectory free linear model predictive control for stable walking in the presence of strong perturbations," in IEEE-RAS International Conference on Humanoid Robots, 2006, pp. 137-142.

[12] O. Stasse, P. Evrard, N. Perrin, N. Mansard, and A. Kheddar, "Fast foot prints re-planning and motion generation during walking in physical human-humanoid interaction," in Humanoid Robots, 2009. Humanoids 2009. 9th IEEE-RAS International Conference on, 2009, pp. 284-289.
[13] O. Kanoun, F. Lamiraux, P.-B. Wieber, F. Kanehiro, E. Yoshida, and J.-P. Laumond, "Prioritizing linear equality and inequality systems: Application to local motion planning for redundant robots," in Proceedings of the 2009 IEEE International Conference on Robotics \& Automation, 2009.

[14] S. Kanzaki, K. Okada, and M. Inaba, "Bracing behavior in humanoid through preview control of impact disturbance," in 5th IEEE-RAS International Conference on Humanoid Robots, 2005, pp. 301-305.

[15] M. Morisawa, F. Kanehiro, K. Kaneko, N. Mansard, J. Sola, E. Yoshida, K. Yokoi, and J.-P. Laumond, "Combining suppression of the disturbance and reactive stepping for recovering balance," in IEEE/RSJ International Conference on Intelligent Robots and Systems, 2010, pp. 3150-3156.

[16] V. Prahlad, G. Dip, and C. Meng-Hwee, "Disturbance rejection by online zmp compensation," in Robotica. Cambridge University Press, 2007, vol. 26, pp. 9-17.

[17] N. Hogan, "Impedance control: An approach to manipulation," in American Control Conference, 1984.

[18] L. Love and W. Book, "Environment estimation for enhanced impedance control," in Robotics and Automation, 1995. Proceedings., 1995 IEEE International Conference on, vol. 2, 1995, pp. 1854 - 1859.

[19] V. Duchaine and C. M. Gosselin, "General model of human-robot cooperation using a novel velocity based variable impedance control," in EuroHaptics Conference, 2007 and Symposium on Haptic Interfaces for Virtual Environment and Teleoperator Systems. World Haptics 2007. Second Joint, 2007, pp. 446 - 451.

[20] O. Khatib, "A unified approach to motion and force control of robot manipulators: The operational space formulation," in IEEE Journal on Robotics \& Automation, vol. 3, no. 1, 1987, pp. 43-53.

[21] J. Szewczyk, F. Plumet, and P. Bidaud, "Planning and controlling cooperating robots through distributed impedance," Journal of Robotic Systems, vol. 19, pp. 283-297, 2002.

[22] J. de Carufel, "Model predictive control of a manipulator arm with frictional/unilateral contact," Ph.D. dissertation, Universit d'Ottawa, 1998.

[23] S. Barthelemy, J. Salini, and A. Micaelli, "Arboris-python." [Online]. Available: https://github.com/salini/arboris-python

[24] J. Dahl and L. Vandenbergue, "cvxopt - python software for convex optimization." [Online]. Available: http://abel.ee.ucla.edu/cvxopt/

[25] G. Sandini, G. Metta, and D. Vernon, "The icub cognitive humanoid robot: An open-system research platform for enactive cognition," in 50 Years of Artificial Intelligence, ser. Lecture Notes in Computer Science. Springer, 2007, ch. 32, pp. 358-369.

[26] J. Nelder and R. Mead, "A simplex method for function minimization," The Computer Journal, vol. 7, pp. 308-313, 1965. 